\title{
Blood traffic control
}

\section{Red blood cell vasodilation: nitric oxide and haemoglobin help to match blood flow to metabolic demand.}

\section{David J. Singel and \\ Jonathan S. Stamler}

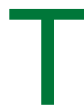
extbook ideas regarding the delivery of oxygen to the body's tissues tend to focus on the cooperativity in the binding and release of oxygen from the transport protein haemoglobin. The uptake of oxygen in the lungs and release of oxygen to the tissues are coupled to structural changes in the haemoglobin tetramer that synergistically alter its affinity for oxygen ligands. Although this mechanism is important, its significance is overemphasized when looking at how oxygen reaches tissues - the main determinant of oxygen delivery is blood flow.

In the microcirculation, blood flow is regulated by physiological oxygen gradients. The progressive decrease in the oxygen content of blood (the oxygen saturation of haemoglobin) as the diameter of the arteriolar blood vessels decreases is coupled to graded vasodilation. A decrease in the oxygen content of blood, in response to (for example) increased muscle activity, stimulates an increase in blood flow. When the demand for oxygen is reduced, the oxygen content of the blood rises and blood flow decreases. Thus, blood flow is precisely matched to metabolic requirements (tissue oxygen consumption). The detailed biochemical mechanism by which oxygen evokes these vascular responses remains unknown.

Arterial blood flow - the trafficking in oxygenated red blood cells - is governed by local modulation of the diameter of small resistance vessels, suggesting a role for the vasodilator nitric oxide (NO). It should be noted that the NO regulation of vessel diameter that resides within the endothelium, and is dependent on shear stress and chemical agonists, is separate from the NO regulation of blood flow that occurs within red blood cells, and is dependent on oxygen. But the sensor of blood oxygen content and the mechanism by which the oxygen signal is transduced to modulate NO bioactivity are unknown. Haemoglobin seems to be an ideal oxygen sensor, but prevailing ideas about the chemistry of its interaction with NO present a conceptual roadblock.

Haemoglobin, in both its oxygenated and deoxygenated forms, was known to react with NO long before there was any awareness of NO's vasoactivity. But as neither of the resulting $\mathrm{NO}$-derived species directly induce vasodilation, it was thought that haemoglobin would only quench NO bioactivity. The discovery of a previously unknown

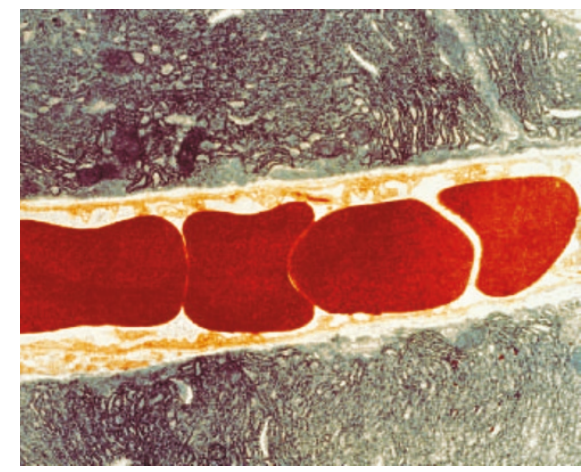

In the flow: red blood cells in the microcirculation.

compound produced with NO, an $S$-nitrosothiol derivative of haemoglobin that has potent vasodilatory activity, changed the picture. Most significantly, this activity is potentiated by the deoxygenation of haemoglobin. Haemoglobin can therefore sense ambient oxygen levels through its oxygenbinding function and adjust NO bioavailability. In turn, red blood cells can cause microvessels to dilate or constrict, with $S$-nitroso-haemoglobin as an intermediate. Thus, the outlines of a mechanistic basis for matching tissue perfusion with metabolic demand were at hand.

Although numerous studies verified the formation of S-nitroso-haemoglobin after $\mathrm{NO} /$ haemoglobin interaction, others found that $\mathrm{NO}$ was bound and rendered inactive by haemoglobin. These divergent results can be understood by considering both the emergent complexity of haemoglobin - well appreciated in the realm of oxygen binding, but rarely considered in its overall $\mathrm{NO}$ biochemistry - and the undervalued importance of physiological conditions. Although the use of excess NO relative to haem was justified in early experiments, where $\mathrm{NO}$ was used primarily as a surrogate for oxygen, it gives a false picture of the physiological situation, in which the ratio is 1 in $1,000-10,000$ and oxygen content is a critical variable.

At physiological concentrations, $\mathrm{NO}$ reacts preferentially with the $\beta$-subunits of haemoglobin (each tetramer contains two $\alpha$-and two $\beta$-subunits), forming $S$-nitroso-haemoglobin in the oxygenated state. In contrast, at supraphysiological levels, NO tends to lodge on the $\alpha$-subunits and favour the deoxygenated state. The disposition and reactivity of $\mathrm{NO}$ bound to haemoglobin is thus a function of multiple variables, including the other allosteric effectors of haemoglobin $(\mathrm{pH}$; partial pressure of carbon dioxide, $\mathrm{pCO}_{2}$; partial pressure of $\mathrm{O}_{2}, \mathrm{pO}_{2}$ ) and the ratio of their concentrations to haem. Changes in conditions can give rise to stark, rather than subtle, linear changes in the distribution of NO reaction products. This complexity emerges both from the cooperative, nonlinear behaviour of the haemoglobin tetramer and from the branched network of coupled kinetic equations underlying this rich chemistry.

How are these ideas relevant to oxygen delivery under physiological conditions? In the blood perfusing tissues, oxygen is in great excess over NO. Haemoglobin is also in excess over other blood oxides of nitrogen with which it interacts (for example, nitrite). Under such conditions, NO binds preferentially to the $\beta$-subunit, with $S$-nitrosothiol formation promoted at higher $\mathrm{pO}_{2}$ and $\mathrm{NO}$ group release promoted at lower $\mathrm{pO}_{2}$. Thus, $S$-nitrosohaemoglobin contributes to the matching of blood flow to demand under physiological conditions. In contrast, when micromolar concentrations of NO arise, as in septic shock, the potential problem of excess $S$-nitrosohaemoglobin and consequent excessive vasodilation is avoided by sequestering $\mathrm{NO}$ on the $\alpha$-haems, which additionally lowers the overall oxygen affinity of the protein. This chemistry restricts NO bioavailability while enhancing oxygen delivery.

A complete kinetic/thermodynamic model of NO's interactions with haemoglobin in the circulation - a respiratory cycle for NO - has yet to be achieved. But an appreciation of the complexity of this problem is essential in designing experiments to elucidate the detailed mechanism by which red blood cells act as oxygen-responsive transducers of NO bioactivity. Emerging evidence that vasodilation by red blood cells is altered in disease, including heart failure, pulmonary hypertension and diabetes, should open a new field of investigation and could potentially change the practice of medicine.

David J. Singel is in the Department of Chemistry and Biochemistry, Montana State University, Bozeman, Montana 59717, USA. Jonathan S. Stamler is at the Howard Hughes Medical Institute, Duke University Medical Center, Durham,

North Carolina 27710, USA.

\section{FURTHER READING}

Duling, B. R. \& Berne, R. M. Circ. Res. 37, 325-322 (1970).

Jia, L. et.al. Nature 380, 221-226 (1996). González-Alonso, J. et al. J. Physiol. 530,

331-341 (2001).

Datta, B. et al. Circulation 109, 1339-1342 (2004). Singel D. J. \& Stamler J. S. Annu. Rev. Physiol. 67, (in the press). 